\title{
Comments on a Floating-Point Version of Nordsieck's Scheme for the Numerical Integration of Differential Equations*
}

\author{
By H. R. Lewis, Jr. and E. J. Stovall, Jr.
}

One of the important problems in numerical analysis which arises in scientific and engineering research is that of numerical integration of a system of differential equations. Because of the frequency with which this problem occurs, it is valuable to have a general-purpose numerical scheme with which the integration of a large class of differential equations can be reliably performed. One numerical scheme which is useful as such a general-purpose method, and well adapted for efficient use with digital computers, has been proposed by A. Nordsieck [1], [2]. This scheme is designed to solve a system of first-order equations,

$$
d y_{i} / d x=f_{i}\left(x, y_{1}, y_{2}, \cdots, y_{n}\right), \quad i=1,2, \cdots, n,
$$

with given initial conditions, whenever the $f_{i}$ are such that a unique solution exists.

The basic formulas of the method are equivalent to the fifth-degree polynomial approximation that can be constructed from the values of the $y_{i}$ and $f_{i}$ at the current value of $x$ along with the values of the $f_{i}$ at the four preceding values of $x$. The equivalent approximating polynomial is identical with that of the Adams method of integration [3], [4]. However, Nordsieck has reformulated and modified the Adams method in a way which is of interest for practical application.

An important practical feature of Nordsieck's scheme is the automatic increase and decrease of the elementary interval size during the course of the integration. This is accomplished by means of two tests which are performed at each elementary integration step. One test adjusts the truncation error in the solution. The other test is intended to guarantee that the integration scheme be numerically stable throughout the integration; that is, those solutions of the equations of the numerical method which are not related to the differential equations are supposed to be damped out if this test is always satisfied. Another important feature of the scheme is that it is self-starting.

Problems which have been done with this method by the authors or their colleagues include integration of the nonrelativistic equations of motion of a charged particle in a magnetic field, integration of the equation for the radial wave function in some problems of atomic physics, ${ }^{1}$ and all of the problems reported by Nordsieck [1]. The method, in its present form, worked very well for all of these problems. Modification of the stability test came as a result of difficulties which were sometimes encountered when integrating the equations of motion of a charged particle

Received October 12, 1964. Revised September 22, 1966.

* Work performed under the auspices of the U. S. Atomic Energy Commission.

${ }^{1}$ For an example of particle trajectory calculations see H. R. Lewis, Jr. and E. J. Stovall, Jr., Bull. Am. Phys. Soc. v. 9, 1964, p. 399; for an example of wave function calculations see R. C. Mjolsness and H. M. Ruppel, Los Alamos Scientific Laboratory Report LA-DC-7713 (to be published in Phys. Rev.). 
with earlier floating-point versions of the integration scheme. These difficulties were (1) reduction of the elementary integration interval to unnecessarily or absurdly small values, and (2) unstable "blow-up" of the solution.

Nordsieck formulated the integration scheme for fixed-point arithmetic. The purpose of this note is to explain modifications of the original formulation which allow satisfactory operation with floating-point arithmetic. ${ }^{2}$ The major modification, discussed in the first section, is a reformulation of the stability test itself, in order to correct a flaw in the original formulation, and this modification is recommended for both fixed-point and floating-point operation. The special procedures proposed by Nordsieck (novel rounding techniques and the use of guard digits), which were helpful in avoiding malfunctions of the test as it was originally formulated, are discussed in the second section.

I. The Stability Test. In his original paper [1] Nordsieck proposes a sufficient condition for insuring stability of the numerical method with a comfortable margin of safety. In terms of the elementary integration interval $h$ and the eigenvalues of the matrix $\partial f / \partial y$, whose elements are $\partial f_{i} / \partial y_{j}$, the condition is $[1 a]$

$$
(95 / 288)|h \lambda| \leqq \frac{1}{8},
$$

for each eigenvalue $\lambda$. Nordsieck does not require satisfaction of this inequality directly. Instead, he proposes a test which is intended to insure satisfaction of the inequality, and which is more easily applied. However, his test does not guarantee the validity of the stability condition (2), except in the special case that only a single differential equation is to be solved. Let $y$ and $f$ denote the column matrices whose elements are $y_{i}$ and $f_{i}$, respectively. In the course of the iterative solution of the implicit equations of the scheme, three approximations to $y$ are computed which are called $y^{(1)}, y^{(2)}$, and $y^{(3)}$. For the present discussion, all we need know about the $y^{(k)}$ is that they are column matrices related to the square matrix $\partial f / \partial y$ through the approximate equation [1b]

$$
\left(y^{(3)}-y^{(2)}\right) \cong(95 / 288) h(\partial f / \partial y)\left(y^{(2)}-y^{(1)}\right) .
$$

If there is only one differential equation to be solved $(n=1)$, then $y^{(1)}, y^{(2)}, y^{(3)}$, and $\partial f / \partial y$ are all single numbers, as opposed to true matrix quantities, and we have

$$
\left|y^{(3)}-y^{(2)}\right| \cong(95 / 288)|h(\partial f / \partial y)|\left|y^{(2)}-y^{(1)}\right| \quad(n=1)
$$

${ }^{2}$ A computer program, incorporating these modifications and using floating-point arithmetic, has been written in the FORTRAN-II and FORTRAN-IV languages and has been found to operate very satisfactorily with the IBM 7094 and 7030 computers. Details of this program are given in Los Alamos Scientific Laboratory Report No. LA-3292 (March 1965), "A FORTRAN Version of Nordsieck's Scheme for the Numerical Integration of Differential Equations"' by H. R. Lewis, Jr. and E. J. Stovall, Jr. The programming details are in large measure based on the computer program of an earlier floating-point version of Nordsieck's integration scheme which was kindly macie available to us by R. M. Brown and P. Ponzo of the Coordinated Science Laboratory of the University of Illinois. That program, dated October 1, 1963 and designated D2 (F) UOFI DEQ, was part of the 1604 computer library of the Coordinated Science Laboratory. The program of another earlier version, dated October 26, 1962 and designated BCC Library Routine No. 5. 02. 06, was made available to us by E. P. Gray and J. G. Monteabaro of the Applied Physics Laboratory of the Johns Hopkins University. 
For the case $n=1$ the test proposed by Nordsieck to insure satisfaction of the stability condition $(2)$ can be written as [1c]

$$
\left|y^{(3)}-y^{(2)}\right| \leqq \frac{1}{8}\left|y^{(2)}-y^{(1)}\right| \text {. }
$$

Indeed, for $n=1$, the inequalities (2) and (5) are identical to within the approximation that (3) is an exact equation. (If $n=1$, then $\lambda=\partial f / \partial y$.)

However, if there are two or more equations to be solved simultaneously $(n>1)$, then the situation is different. Let a norm of a matrix be denoted by enclosing the matrix symbol between double vertical bars; then, with suitably chosen norms, the relation corresponding to Eq. (4) is ${ }^{3}$

$$
\frac{95}{288}\left\|h \frac{\partial f}{\partial y}\right\| \gtrsim \frac{\left\|y^{(3)}-y^{(2)}\right\|}{\left\|y^{(2)}-y^{(1)}\right\|} \quad(n>1) .
$$

Let $\lambda_{\max }$ be that eigenvalue of $\partial f / \partial y$ which has the largest magnitude. A standard inequality relating $\|\partial f / \partial y\|$ and $\left|\lambda_{\max }\right|$ is [5]

$$
\left|\lambda_{\max }\right| \leqq\|\partial f / \partial y\| \text {. }
$$

The stability test proposed by Nordsieck for the case $n>1$ may be written as [1c]

$$
\left\|y^{(3)}-y^{(2)}\right\| /\left\|y^{(2)}-y^{(1)}\right\| \leqq \frac{1}{8},
$$

where the column matrix norm used is either the largest magnitude of any element, or the euclidean norm. From relations $(6),(7)$ and (8), we see that no bound whatsoever is obtained on $\|h \partial f / \partial y\|$ or on $\left|h \lambda_{\max }\right|$. Indeed, there are cases for which the inequality (8) which represents the test is satisfied while the inequality (2) which represents the stability condition is violated. ${ }^{4}$ Numerical examples illustrating this point can be found easily.

The stability test which we propose, and which we have used in our floatingpoint version of the scheme, is precisely the basic stability condition given by expression (2). That is, the elements of the matrix $\partial f / \partial y$ are evaluated-analytically if possible, numerically otherwise-and either an upper bound of the magnitudes of the eigenvalues, or the largest eigenvalue itself, is computed and used in (2).

The original stability test was subject to malfunction because of round-off noise. This difficulty was alleviated, in the fixed-point version, by use of novel rounding techniques and by use of guard digits. Such round-off noise problems do not interfere with the direct application of the stability condition.

II. Guard Digits and Special Rounding Procedures. In the fixed-point version of this integration scheme, with the stability test in the form given by expression (8), Nordsieck found it desirable to carry $\log _{\beta}\left(|h|^{-1}\right)$ more digits in $y$ than in $f$-so-called guard digits $[1 d]$. ( $\beta$ is the base of the number system with which computations are performed. For example, $\beta=2$ with binary arithmetic.) The reason given for this is to minimize the accumulation of round-off error in $y$ when the

${ }^{3}$ If the square matrix and column matrix norms are so chosen that (6) is valid, then these norms are said to be consistent with one another. Norms are usually chosen in this way. See, for example, [5].

${ }^{4} \mathrm{~A}$. Nordsieck (private communication) agrees that the stability test as originally formulated does not insure satisfaction of the stability condition (2). 
number of elementary steps is large. A different reason for keeping the guard digits is that a certain form of round-off noise then tends not to interfere with the functioning of the original stability test (8). This can be seen in the following way. From the equations of the integration scheme [1], it is easily derived that the differences, $\left(y^{(3)}-y^{(2)}\right)$ and $\left(y^{(2)}-y^{(1)}\right)$, can be expressed as

$$
y^{(3)}-y^{(2)}=(95 h / 288)\left\{f\left[x+h, y^{(2)}(x+h)\right]-f\left[x+h, y^{(1)}(x+h)\right]\right\}
$$

and

$$
y^{(2)}-y^{(1)}=(95 h / 288)\left\{f\left[x+h, y^{(1)}(x+h)\right]-f^{p}\right\} .
$$

The $y^{(i)}$ are approximations to $y$ for the independent variable equal to $x+h ; f^{p}$, called the "predicted" value of $f$ in [1], is a first approximation to the value of $f$ at $x+h$. Using Eqs. (9a) and (9b), the original stability test (8) can be rewritten as

$$
\begin{aligned}
&\left\|f\left[x+h, y^{(2)}(x+h)\right]-f\left[x+h, y^{(1)}(x+h)\right]\right\| \\
& \leqq \frac{1}{8}\left\|f\left[x+h, y^{(1)}(x+h)\right]-f^{p}\right\| .
\end{aligned}
$$

Since the inequality depends explicitly on differences of computed values of the derivatives, it is evident that round-off noise in those values can interfere with the functioning of the original stability test. We can estimate the amount of error in $f(x, y)$ due to an error in $y$, for the case $n=1$, as follows. Letting $\Delta y$ be the error in $y$ and $\Delta f$ the corresponding error in $f$, we have, in first approximation,

$$
|\Delta f| \cong|\partial f / \partial y||\Delta y| \text {. }
$$

However, $|\partial f / \partial y|$ is bounded by the stability condition

$$
\left|\frac{\partial f}{\partial y}\right| \leqq \frac{1}{8(95 / 288)|h|} .
$$

Combining (11) and (12), we have

$$
|\Delta f| \lesssim \frac{36}{95}\left|\frac{1}{h}\right||\Delta y|=\beta^{\log _{\beta}(36 / 95 \cdot 1 / / h \mid)} \Delta y .
$$

Taking the case of binary arithmetic $(\beta=2)$, we see that, for $n=1$, an error equal to the least count in $y$ will usually give rise to an error less than the least count in $f$ if $\log _{\beta}(1 /|h|)$ more digits are carried in $y$ than in $f$. Thus, round-off noise of the magnitude of the least count in $y$ would tend not to interfere with application of a stability test in the form of expression (10).

In floating-point arithmetic it is difficult to use guard digits for stopping the propagation of round-off noise from $y$ into $f$, even in the case $n=1$. (One possibility, which is numerically not quite equivalent to the use of guard digits in fixed-point arithmetic, is to use double-precision arithmetic for $y$.) It is, therefore, fortunate that the present stability test, which is identical to the stability condition (2), is not influenced by such noise. Neither guard digits nor double-precision arithmetic have been used in our present floating-point version of Nordsieck's scheme.

A novel way of rounding certain quantities which appear in this integration scheme was introduced in the original fixed-point version; this type of rounding was 
called "rounding away from zero" [10]. The purpose of this rounding was to eliminate a type of noise which sometimes interfered with the proper operation of the tests which control the size of the elementary interval. A floating-point procedure can be devised which is analogous to rounding away from zero in fixed-point. We have tried this procedure in our floating-point version of the integration scheme which incorporates the new stability test. However, with that version of the integration scheme, we have not observed any over-all improvement in the operation of the interval control logic when these rounding procedures are included; nor have we observed any malfunction of the interval control logic when these special rounding techniques are omitted. The floating-point "rounding away from zero," at least when done with the Fortran computer language, is rather time consuming, with the result that the computer time necessary for a particular problem can be substantially longer with the special rounding techniques than without. For these reasons, "rounding away from zero" has been omitted from the floating-point version of the integration scheme.

We express our appreciation to R. M. Brown and P. Ponzo of the Coordinated Science Laboratory of the University of Illinois, and to E. P. Gray and J. G. Monteabaro of the Applied Physics Laboratory of the Johns Hopkins University, for kindly providing us with their floating-point versions of Nordsieck's integration scheme. The present work began as a result of using their computer programs.

Los Alamos Scientific Laboratory, University of California

Los Alamos, New Mexico

1. A. Nondsieck, "On numerical integration of ordinary differential equations," Math. Comp., v. 16, 1962, pp. 22-49. MR 24 * B2552.

a) Equation (8) and pp. 29, 30 and 36 .

b) Equations (8) and (20).

c) Equation (22a) and p. 36 .

d) pp. 25,35 and 46 .

e) pp. 38-39 and Appendix C.

2. A. Nordsieck "Automatic numerical integration of ordinary differential equations," Proc. Sympos. Appl. Math., Vol. 15, pp. 241-250, Amer. Math. Soc., Providence, R. I., 1963. MR 28 * 1771.

3. W. E. Milne, Numerical Solution of Differential Equations, Wiley, New York and Chapman \& Hall, London, 1953, pp. 53-55. MR 16, 864.

4. L. Collatz, The Numerical Treatment of Differential Equations, Die Grundlehren der mathematischen Wissenschaf ten, Band 60, Springer, Berlin, 1960, pp. 83-86. MR 22 *322.

5. J. H. Wilk Inson, Rounding Errors in Algebraic Processes, Her Majesty's Stationery Office, London and Prentice-Hall, Englewood Cliffs, N. J., 1963, pp. 79-82. MR 28 *4661. 